\title{
Phase-locking regions in a forced model of slow insulin and glucose oscillations
}

Sturis, Jeppe; Knudsen, Carsten; O'Meara, Niall M.; Thomsen, Jesper Skovhus; Mosekilde, Erik; Cauter, Eve Van; Polonsky, Kenneth S.

\section{Published in:}

Chaos

Link to article, DOI:

$10.1063 / 1.166068$

Publication date:

1995

Document Version

Publisher's PDF, also known as Version of record

Link back to DTU Orbit

Citation (APA):

Sturis, J., Knudsen, C., O'Meara, N. M., Thomsen, J. S., Mosekilde, E., Cauter, E. V., \& Polonsky, K. S. (1995). Phase-locking regions in a forced model of slow insulin and glucose oscillations. Chaos, 5(1), 193-199. https://doi.org/10.1063/1.166068

\section{General rights}

Copyright and moral rights for the publications made accessible in the public portal are retained by the authors and/or other copyright owners and it is a condition of accessing publications that users recognise and abide by the legal requirements associated with these rights.

- Users may download and print one copy of any publication from the public portal for the purpose of private study or research.

- You may not further distribute the material or use it for any profit-making activity or commercial gain

- You may freely distribute the URL identifying the publication in the public portal 


\title{
Phase-locking regions in a forced model of slow insulin and glucose oscillations
}

\author{
Jeppe Sturis \\ Department of Medicine, The University of Chicago, Chicago, Illinois 60637 and Physics Department, \\ The Technical University of Denmark, DK-2800 Lyngby, Denmark \\ Carsten Knudsen \\ Physics Department, The Technical University of Denmark, DK-2800 Lyngby, Denmark \\ Niall M. O'Meara \\ Department of Medicine, The University of Chicago, Chicago, Illinois 60637 \\ Jesper S. Thomsen and Erik Mosekilde \\ Physics Department, The Technical University of Denmark, DK-2800 Lyngby, Denmark \\ Eve Van Cauter and Kenneth S. Polonsky \\ Department of Medicine, The University of Chicago, Chicago, Illinois 60637
}

(Received 8 December 1992; accepted for publication 8 August 1994)

\begin{abstract}
We present a detailed numerical investigation of the phase-locking regions in a forced model of slow oscillations in human insulin secretion and blood glucose concentration. The bifurcation structures of period $2 \pi$ and $4 \pi$ tongues are mapped out and found to be qualitatively identical to those of several other periodically forced self-oscillating systems operating across a Hopf-bifurcation point. The numerical analyses are supplemented by clinical experiments. (C) 1995 American Institute of Physics.
\end{abstract}

\section{INTRODUCTION}

Phase-locking and other nonlinear dynamic phenomena have been observed in virtually all areas of science and engineering. Human physiology is an example of a field in which an abundance of self-oscillatory subsystems exist, allowing for a variety of nonlinear dynamic phenomena to arise. The present paper deals with an example of such a system, namely the insulin/glucose feedback mechanism in the human body.

The main function of the hormone insulin is to regulate the uptake of glucose by muscle and other cells, thereby controlling the blood glucose concentration. The most important regulator of pancreatic insulin secretion is glucose, and thus insulin and glucose are the two main components of a negative feedback system. In normal humans, ultradian oscillations with an approximate 2 -hour period can be observed in pancreatic insulin secretion and blood glucose concentration. ${ }^{1-3}$ In addition, more rapid 8-15 min pulses of secretion have also been identified. ${ }^{4}$ The two modes of oscillations are clearly distinct phenomena, ${ }^{1}$ and it appears that they have different origins. The persistence of rapid pulses in the perfused pancreas ${ }^{5}$ and in isolated islets ${ }^{6}$ suggests that they are controlled by an intrapancreatic pacemaker mechanism. Because of their relatively high frequency, they are of fairly low amplitude in the systemic circulation of humans. In contrast, the ultradian oscillations are of large amplitude in peripheral blood, especially when insulin secretion is stimulated by glucose administration, and their origin may well be the insulin/glucose feedback system, i.e., the wholebody feedback regulation of glucose and insulin is unstable. We have previously proposed a nonlinear dynamic model of the insulin/glucose feedback system, ${ }^{7}$ The model exhibits self-sustained ultradian oscillations when a constant glucose infusion experiment is simulated.

Additionally, we have $\operatorname{shown}^{8}$ how the ultradian oscillations in insulin secretion, which persist during constant intravenous glucose infusion, can be entrained to the pattern of an oscillatory glucose infusion. When glucose is infused as a sine wave with a relative amplitude of $33 \%$ and a period either $20 \%$ below or $20 \%$ above the period occurring during constant glucose infusion, the oscillations in glucose concentration and insulin secretion entrain to the pattern of glucose infusion. There are limits to the range within which entrainment can be obtained. Thus, if the period of the oscillatory glucose infusion is outside a certain range, simple 1:1 entrainment can no longer be obtained. In Fig. 1, the result of an experiment first reported in Ref. 8 is shown. Here, the period of infusion was approximately twice as long as the period observed when a constant infusion of glucose was administered, and as a. result, two large amplitude oscillations appear for every swing of the glucose infusion. These modes of entrainment can also be accounted for by the model if the simulated pattern of glucose infusion is oscillatory $^{9}$ as exemplified in Fig. 1. Other experimental evidence of nonlinear dynamic phenomena in periodically forced physiological systems include phase-locking and global bifurcations in periodically forced chick heart cells, ${ }^{10}$ phase-locking in mechanically ventilated cat respiratory systems, ${ }^{11}$ and chaos and phase-locking in periodically stimulated squid axons. ${ }^{\text {i2: }}$

In this paper, we extend the experimental evidence of the insulin and glucose oscillations and the entrainment phenomenon by reporting the results of six additional experiments and comparing them to simulations with the model. Subsequently, we move on to a numerical investigation, mapping 

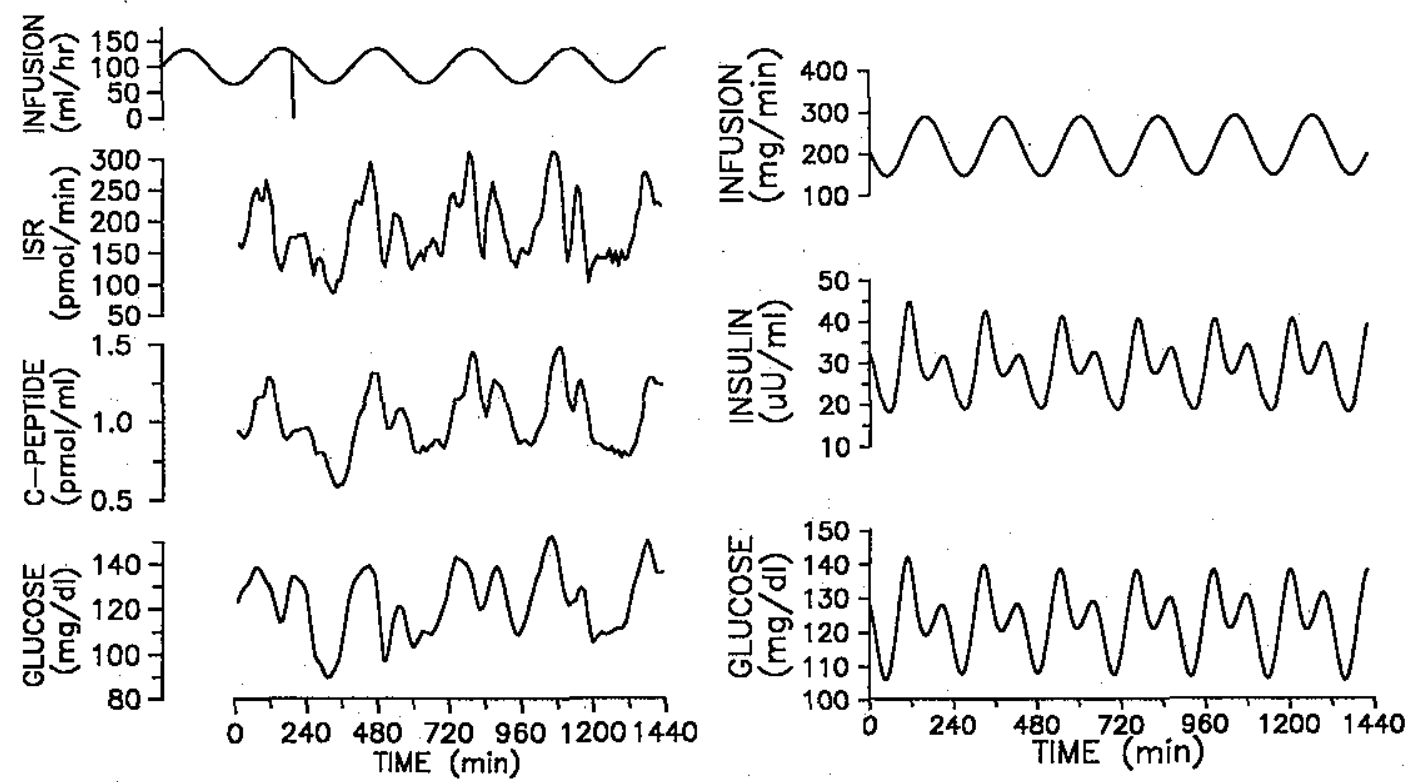

FIG. 1. Left: Glucose infusion study performed in subject A. Or this occasion, the period of the glucose infusion was 320 min, and consequently, there were two large amplitude oscillations in glucose, insulin, and insulin secretion rate (ISR) for each oscillation of the infusion. The vertical line in the infusion curve represents a short, accidental interruption in the glucose infusion which was monitored. Redrawn from Ref. 8. Right: Model simulation in which the period of infusion was approximately twice the endogenous period, and a 2:1 type entrainment is produced.

out some of the largest phase-locking regions of the forced model.

\section{DESIGN OF CLINICAL EXPERIMENTS}

Experimental protocol. Two studies were performed on separate occasions in each of three non-diabetic men (subjects $A, B$ and $C$; ages 27, 21, and 30 yr; weights 54,96 , and $67 \mathrm{~kg}$; heights 169,175 , and $171 \mathrm{~cm}$ ). Subject A had been previously studied. ${ }^{8}$ After an overnight fast, each subject was admitted to the Clinical Research Center at the University of Chicago. An intravenous catheter was inserted into each arm, one for blood sampling, and one for glucose infusion. Beginning at 0800 hours, glucose $(20 \%$ dextrose) was administered for 28 hours via a computer-controlled pump (Flo-gard 8000 volumetric infusion pump; Travenol Laboratories, Deerfield, IL). Sampling for glucose, insulin, and C-peptide was performed at $10-\mathrm{min}$ intervals for the final 24 hours of the study. The mean rate of glucose infusion was $6 \mathrm{mg} /(\mathrm{kg}$ body weight $/ \mathrm{min}$. For subject $\mathrm{A}$, the pattern of infusion was a sine wave with amplitude and period of $40 \%$ and $300 \mathrm{~min}$, and $33 \%$ and $78 \mathrm{~min}$ in the two studies, respectively. The hypothesis of the first experiment was that another example of $2: 1$ entrainment would emerge, [i.e., two oscillations in glucose, insulin, and insulin secretion rate (ISR) for every infusion period] while the second study was performed in an attempt to produce a case 1:2 entrainment (i.e., one full oscillation in glucose, insulin, and ISR for every two oscillations in the infusion). Subjects $\mathrm{B}$ and $\mathrm{C}$ both first received constant glucose infusion, and subsequently an oscillatory glucose infusion with a $33 \%$ amplitude and periods of 60 min (subject B) and 480 min (subject C), respectively.

Glucose, insulin, and C-peptide assays. Glucose concentrations were measured with the glucose oxidase technique using a YSI analyzer (Yellow Springs Instrument Co., Yellow Springs, $\mathrm{OH}$ ). Serum insulin and plasma $\mathrm{C}$-peptide were measured by radioimmunoassay. ${ }^{13,14}$

Data analysis. The raw data were smoothed with a threepoint moving average. ISRs were calculated by deconvolution of the smoothed C-peptide data using a twocompartmental model of C-peptide kinetics. ${ }^{15,16}$ The kinetic parameters of subject A were calculated in connection with the prior set of experiments ${ }^{8}$ while standard parameters were used for subjects $B$ and $C .^{17}$.

\section{THE MODEL OF THE INSULIN/GLUCOSE FEEDBACK MECHANISM}

A detailed description of the model has previously been published. ${ }^{7}$ The model was constructed by extracting parameters and qualitative and quantitative features of functional relationships from the results of independent clinical experiments. The model equations are:

$$
\begin{aligned}
& \frac{d I_{p}}{d t}=f_{1}(G)-E\left(\frac{I_{p}}{V_{p}}-\frac{I_{i}}{V_{i}}\right)-\frac{I_{p}}{\tau_{p}}, \quad \frac{d I_{i}}{d t}=E\left(\frac{I_{p}}{V_{p}}-\frac{I_{i}}{V_{i}}\right)-\frac{I_{i}}{\tau_{i}}, \\
& \frac{d G}{d t}=G I\left(1+A \sin \left(\frac{2 \pi t}{P}\right)\right)-f_{2}(G)-f_{3}(G) f_{4}\left(I_{i}\right)+f_{5}\left(x_{3}\right), \\
& \frac{d x_{1}}{d t}=\frac{I_{p}-x_{1}}{\tau_{d} / 3}, \quad \frac{d x_{2}}{d t}=\frac{x_{1}-x_{2}}{\tau_{d} / 3}, \quad \frac{d x_{3}}{d t}=\frac{x_{2}-x_{3}}{\tau_{d} / 3},
\end{aligned}
$$

where the functional relationships and base case parameters are:

$$
\begin{aligned}
& f_{1}=\frac{209}{1+\exp \left(-G /\left(300 V_{g}\right)+6.6\right)}, \\
& f_{2}=72\left[1-\exp \left(-G /\left(144 V_{g}\right)\right)\right],
\end{aligned}
$$



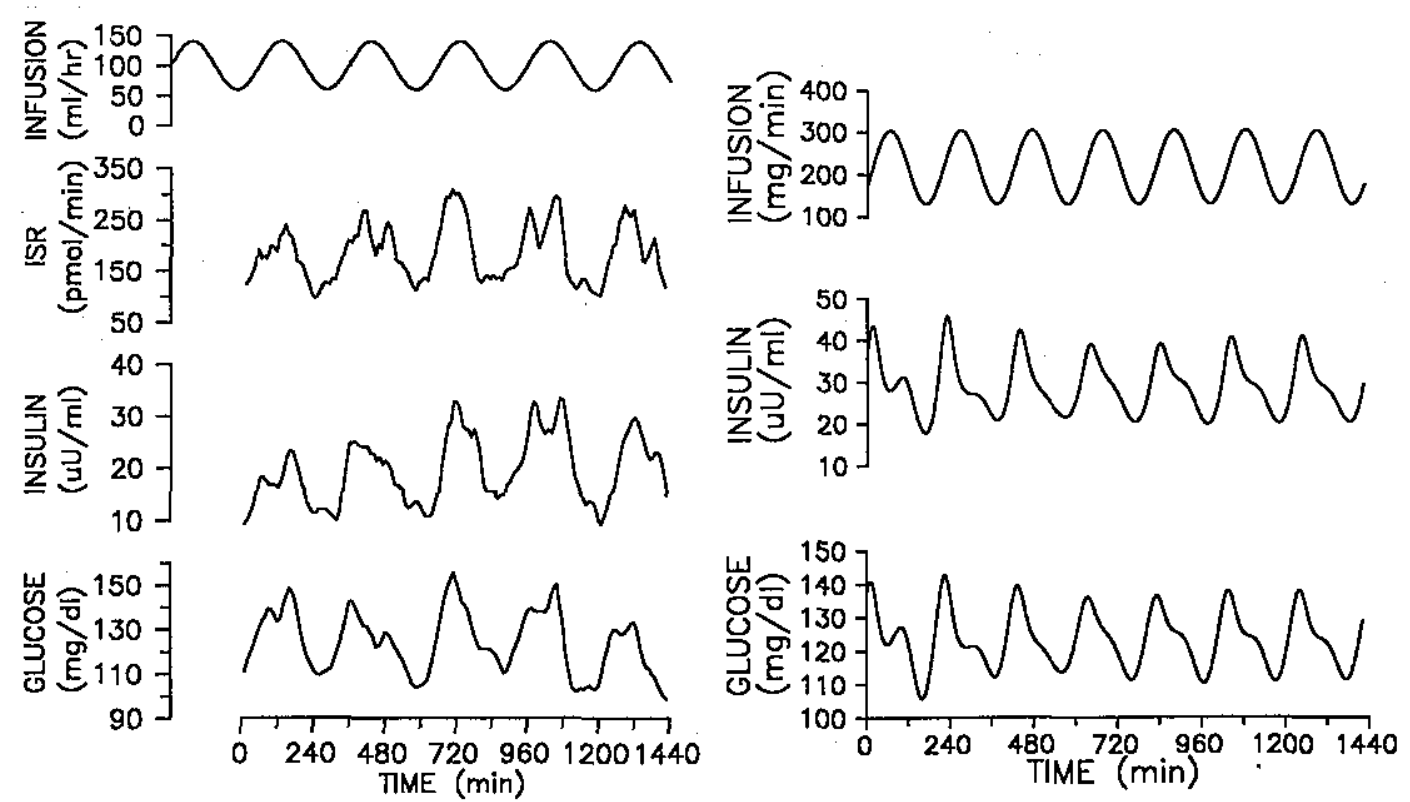

FIG. 2. Left: Clinical experiment in subject $A$ with a period of $300 \mathrm{~min}$ and an amplitude of $\pm 40 \%$ for the glucose infusion. In this case, the $2: 1$ entrainment observed in Fig. 1 appears to be lost, in that the second peak is replaced by a "shoulder" in the profiles in some cases and absent in other cases. Right: Corresponding simulation in which the pcriod of infusion was reduced by $8 \%$ and the amplitude was increased to $40 \%$.

$f_{3}=0.01 G / V_{g}$,

$f_{4}=\frac{90}{1+\exp \left(-1.772 \log \left(I_{i}\left[1 / V_{i}+1 /\left(E \tau_{i}\right)\right]\right)+7.76\right)}+4$,

$f_{5}=\frac{180}{\left(1+\exp \left(0.29 x_{3} / V_{p}-7.5\right)\right)}$;

$V_{p}=3 \mathrm{~L}, V_{i}=11 \mathrm{~L}, V_{g}=10 \mathrm{~L}, \tau_{p}=6 \mathrm{~min}, \tau_{i}=100 \mathrm{~min}$, $\tau_{d}=36 \mathrm{~min}, E=0.2 \mathrm{~L} / \mathrm{min}$, and $G I=216 \mathrm{mg} / \mathrm{min}$.

Insulin secretion is stimulated by glucose $\left(f_{l}\right)$. After its secretion by the pancreas, insulin distributes in the plasma space with a volume of $V_{p}$ and subsequently it is either degraded according to time constant $\tau_{p}$ or it enters the interstitial fluid with a volume of $V_{i}$ by diffusion with an effective diffusion constant $E$. Interstitial insulin, which is degraded slowly $\left(\tau_{i}\right)$, enhances glucose utilization $\left(f_{4}\right)$. Additionally, glucose-which is assumed to distribute in one compartment with volume $V_{g}$-also enhances its own utilization $\left(f_{2}\right.$, insulin independent uptake, and $f_{3}$, insulin dependent uptake). Insulin also inhibits hepatic glucose production $\left(f_{5}\right)$, but there is a time delay between the appearance of insulin in the plasma and its resulting suppressive effect. The pathway of this time delay is presently unknown and therefore it is modeled as a third-order delay with a lag time $\tau_{d \cdot}$. Glucose is infused as a sine wave, mean rate $G l$, amplitude $A$, and period $P$. These three parameters can be varied in the experimental setting. In the following section, we will present numerical simulations as well as perform a two-parameter bifurcation analysis of the forced insulin/glucose model by varying the amplitude and period of the oscillatory glucose infusion while other model parameters are fixed.

\section{SIMULATION AND EXPERIMENTAL RESULTS}

In accordance with typical experimental results, simulation of a constant glucose infusion yields self-sustained oscillations of insulin and glucose. ${ }^{7}$ The period of the oscillation is approximately $110 \mathrm{~min}$, very close to the experimentally observed period. By simulating oscillatory glucose infusions, entrainment of the insulin and glucose oscillations occurs, providing the forcing period is within a certain range of the natural period. ${ }^{9}$ These observations are in complete agreement with our previous experimental results. ${ }^{8}$ If the period of infusion is approximately twice that of the endogenous period, a mode of entrainment will be obtained in which the system exhibits two large amplitude oscillations for each period of the forcing. This is also in harmony with our experimental results. The region in which this particular type of entrainment is possible is, however, narrow. When the period of infusion was set at $300 \mathrm{~min}$ instead of $320 \mathrm{~min}$ and the forcing amplitude increased from $33 \%$ to $40 \%$, the result was qualitatively different in that only one large amplitude peak of plasma glucose and insulin secretion could be observed. This experimental result is shown in the left panel of Fig. 2. For comparison, a simulation was performed with a $40 \%$ forcing amplitude and a forcing period $8 \%$ shorter than the period used for the simulation shown in the right panel of Fig. 1. The result displayed in the right panel of Fig. 2 is similar to the experimental result.

If the infusion period is approximately half that of the natural period of the system, one would expect a 1:2 type of entrainment, i.e., the periodic forcing goes through two oscillations before the system has completed one full cycle. The result of the experiment in which the period of infusion was $78 \mathrm{~min}$ is shown in the left part of Fig. 3. During the first 


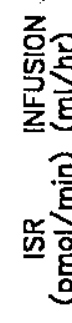

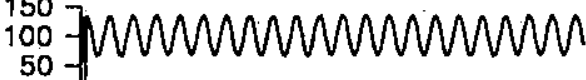
o
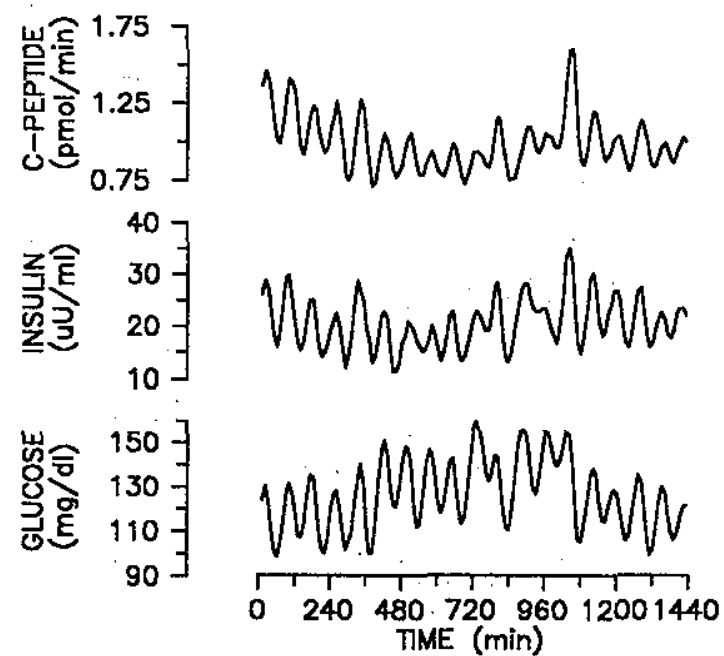
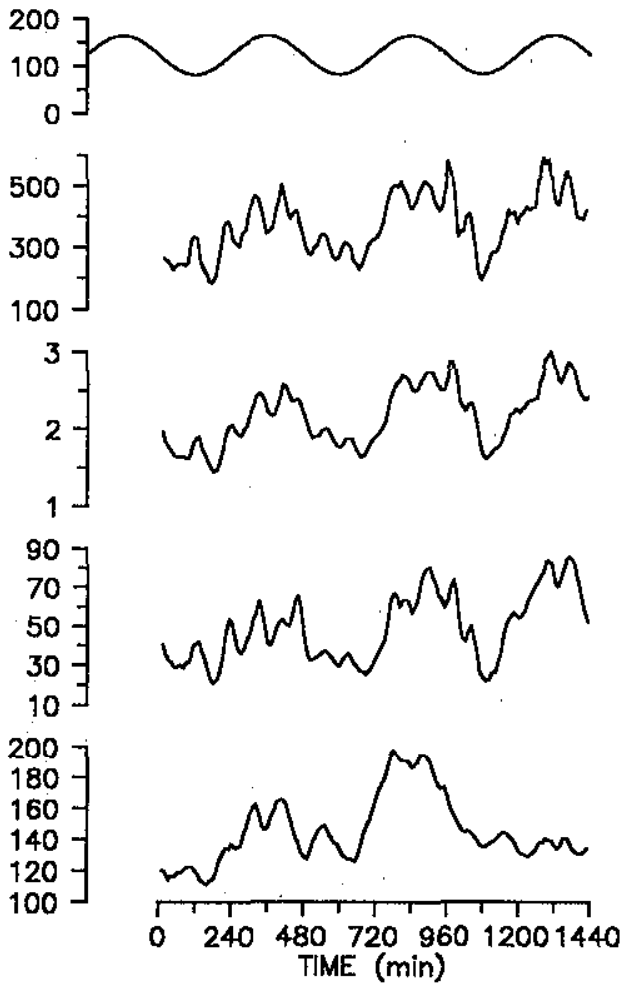

FIG. 3. Left: Clinical experiment in subject $A$ with a period of $78 \mathrm{~min}$ and an amplitude of $\pm 33 \%$ for the glucose infusion. During the first 16 hours of the study, the mode of oscillation resembles entrainment in a 1:2 fashion in that the troughs in the glucose profile alternate between higher and lower values, After 16 hours, this mode is lost, but it reappears towards the end of the study. Right: Clinical experiment in subject $\mathrm{C}$ with a period of 480 min and an amplitude of $\pm 33 \%$ for the glucose infusion.

16 hours of the study, the system shows some signs of being entrained in the expected fashion: the troughs of glucose alternate for each infusion cycle. A similar pattern is difficult to discern for the ISR and insulin profiles. After approximately 16 hours, the system loses any sign of 1:2 entrainment, but towards the end of the experiment, the mode reappears. An analogous simulation result (not shown) shows clear 1:2 critrainment. During constant glucose infusion, ultradian oscillations in glucose and insulin secretion occurred in both subjects $B$ and $C$ with an approximate period of 120 min (data not shown). In subject B, during oscillatory glucose infusion with a period of $60 \mathrm{~min}$, the expected 1:2 entrainment pattern did not clearly emerge. Instead, one oscillation in glucose and insulin secretion was evident for each oscillation of the infusion (data not shown), a result very. similar to the one obtained in subject A (Fig. 3, left). In subject $\mathrm{C}$, during oscillatory glucose infusion with a period of $480 \mathrm{~min}$, the resulting glucose and insulin secretory profiles revealed 3-5 oscillations for every one oscillation of the infusion (Fig. 3, right). Although one might have expected to observe 4:1 entrainment, the experimental result more closely resembles nonperiodic behavior, e.g., quasiperiodic oscillations.

\section{BIFURCATION ANALYSIS OF ENTRAINMENT REGIONS IN THE MODEL.}

\section{A. Numerical methods}

We have used the software package PATH ${ }^{18}$ which can locate stable as well as unstable solutions by use of a Newton iteration scheme. The stability of a periodic orbit is determined by calculating the eigenvalues of the derivative of the Poincare map. A bifurcation point can be located by varying one or two parameters slowly. Once a bifurcation point is found, simultaneous and slow variation of two parameters can automatically be performed by PATH, allowing the curve of bifurcation points which generically exists in a twoparameter case to be traced.

\section{B. Bifurcation analysis of the unforced system}

Before analyzing the forced system, it is appropriate to perform some investigation of the autonomous insulin/ glucose model. A two-parameter bifurcation diagram is shown in the inset of Fig. 4. Here the rate of glucose infusion $G I$ and the volume $V_{p}$ of plasma which insulin first enters are the bifurcation parameters. The system is stable for both low and high values of $G I$ and $V_{p}$. In the region of intermediate values for these parameters, a stable limit cycle solution exists along with the unstable equilibrium. The boundary between these two regions is a curve of supercritical Hopfbifurcation points. In the analysis of the forced system, GI is fixed at $216 \mathrm{mg} / \mathrm{min}$ and $V_{p}$ at $3 \mathrm{~L}$. Since the periodic forcing influences the size of $G I$, a sufficiently large amplitude of the periodic variation will cause $G I$ to take on values for which the autonomous system is stable.

\section{Structure of dominant Arnol'd tongues}

Figure 4 also presents a phase diagram illustrating all the bifurcation curves which were found in the present numeri- 


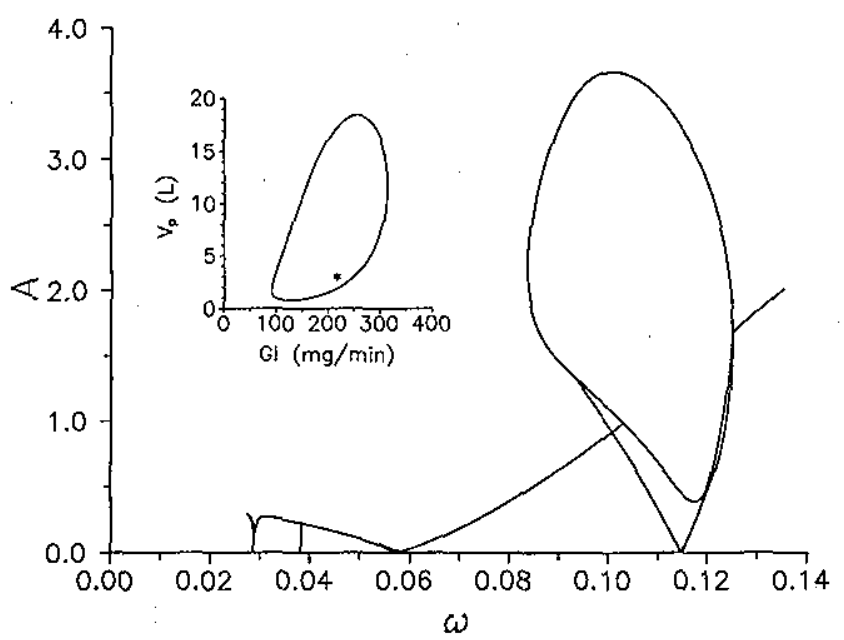

FIG. 4. The inset shows a bifurcation diagram in the $V_{p}-G l$ plane of the autonomous model; the closed curve represents supercritical Hopfbifurcations and the asterisk illustrates the parameter combination for the base case simulation. The rest of the figure shows a phase diagram presenting an overview of all the bifurcation curves found in the analysis of the forced model.

cal analysis. Our analysis was confined to regions for which the period of the phase-locked solution was either equal to or twice that of the periodic forcing. In addition to the local bifurcation curves shown in the figure, we have also found evidence of global bifurcations involving chaotic transitions and associated with the codimension 2 bifurcation points, some of which are shown in Fig. 5.

Period $2 \pi$ regions. The top panel of Fig. 5 shows a magnified region of the tongue for which the forcing period is close to that of the autonomous system. For the lowest amplitudes, both tongue edges are saddle-node bifurcations. As the amplitude increases, both sides of the tongue become torus bifurcation (also denoted Neimark-Sacker bifurcation) curves. The left hand side of the saddle-node edge begins to overlap with the torus edge, turns sharply in a cusp-point, and subsequently connects to the torus part of the edge. At this connection point, the saddle-(stable)node bifurcation becomes a saddle-(unstable)node bifurcation, and the curve continues until it joins the right hand torus edge, at which point the bifurcation again is a saddle-(stable)node bifurcation, and the curve almost simultaneously turns and constitutes the lower right hand side of the tongue. The tongue for which the forcing period is approximately twice that of the period of the autonomous system has the identical qualitative structure as the tongue shown in the top of Fig. 5. Because the period of the steady state solutions in these tongues is equal to the period of the forcing, they are both denoted $2 \pi$ regions. For low forcing amplitudes, however, in the latter tongue there are almost two full swings in the state variables for each swing of the forcing, but the system only performs a complete cycle with two swings of the forcing. As the amplitude is increased and the period of the forcing differs significantly from twice the period of the autonomous system, the shape of the limit cycle changes so that only one peak in the state variables can be observed. This transition between a solution in which two peaks can be seen in each period to a
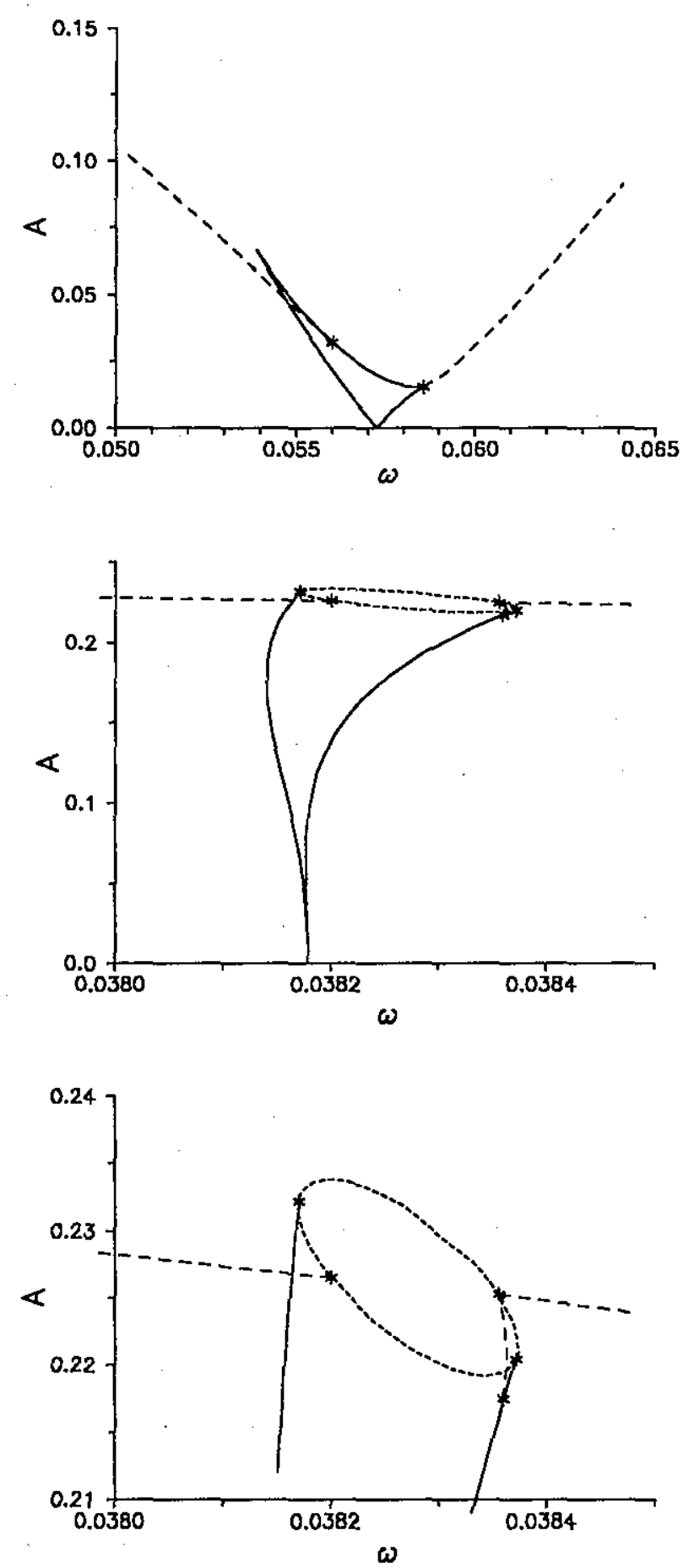

FIG. 5. Top panel: Local bifurcation curves in period $2 \pi$ region for which the period of the forcing is close to the period of the autonomous system. Middle panel: Local bifurcation curves in period $4 \pi$ regions for which the forcing period is approximately 1.5 times the endogenous period. Bottom panel: Magnification of the region shown in the middle panel. Saddle-node bifurcations are full curves, period-doubling bifurcations are short-dashed curves and torus bifurcations are long-dashed curves. The asterisks denote connections of bifurcation curves-codimension-2 bifurcation points.

solution in which only one peak can be seen (see also Figs. 1 and 2) does not involve a bifurcation, but merely a continuous change in the shape of the limit cycle.

Period $4 \pi$ tongues. The middle and bottom panels of Fig. 5 illustrate the $4 \pi$ tongue emerging from the $\omega$-axis at 
two swings of the periodic forcing for every three oscillations of the autonomous system. Although this region is much smaller than the $4 \pi$ tongue emerging from the $\omega$-axis at a forcing period equal to half the period of the autonomous system (see full phase diagram in Fig. 4), both regions involve period-doubling, saddle-node and torus bifurcation curves. In particular, the way the curves join appears to be qualitatively identical in the two regions.

\section{DISCUSSION}

The present analysis represents an effort to bridge a gap between the field of clinical experimental research and nonlinear dynamics. Our research design was specifically aimed at explaining each individual experimental temporal profile. From the outset, it was assumed that the insulin/glucose feedback regulation is of importance for the genesis and control of the slow, self-sustained oscillations which can be observed in normal man. Administration of oscillatory glucose infusions enabled various modes of entrainment and quasiperiodic behavior to be observed, complementing our previous experimental results. ${ }^{8}$

The agreement between the experimental and simulation results is by no means complete. It was particularly difficult to produce an experimental example of 1:2 entrainment, i.e., one full cycle of the system behavior for every two oscillations in the infusion. One of the most likely explanations for this is the fact that many other self-oscillatory processes exist within the body which influence the insulin-glucose feedback mechanism. For example, the circadian clock and the sleepwake cycle have both been shown to have independent and strong effects on glucose regulation, ${ }^{19}$ and the pulsatile secretion of hormones such as luteinizing hormone $(\mathrm{LH})$ and growth hormone $(\mathrm{GH})$ may also influence the temporal profiles of glucose and insulin secretion. We have shown that this is indeed the case for GH. ${ }^{20}$ Since certain features appear to be well-represented by the model, the subsequent numerical investigation serves as an illustration of how the entrainment regions in the experimental system could be structured, but it is not a prediction of exact locations of bifurcation curves in the experimental setting.

A potential advantage of the present experimental approach lies in the improved signal to noise ratio which is a consequence of the increased amplitude of the secretory oscillations due to the periodic forcing. This aspect could facilitate a closer investigation of how other hormones might phase-lock or interact with the insulin/glucose oscillations.

We have recently shown that the pattern of $1: 1$ entrainment observed during oscillatory glucose infusion in nondiabetic subjects is absent in patients with Type 2 (non-insulindependent) diabetes mellitus and even in subjects with only a mild impairment of glucose tolerance. ${ }^{21}$ In its present form, those experimental results cannot be reproduced by the mathematical model of the insulin-glucose feedback mechanism. One of the obvious shortcomings of the model is the simple relationship that has been assumed to exist between glucose and insulin secretion. This relationship does not accurately represent the in vivo insulin secretory response in a diabetic person. Further development of the model would be useful in elucidating the experimental results observed in diabetes.

A large part of Fig. 4 deals with a region of parameter space which is physiologically unrealistic. Values of $A$ larger than 1 are equivalent to pumping glucose out of instead of into a vein. While such a situation would be impossible to realize experimentally, it is of theoretical interest, and the numerical analyses show that bifurcation phenomena may occur in the unrealistic region of parameter space that are important for the dynamics in the realistic region. For instance, the codimension- 2 bifurcation point joining the torus and period-doubling curves in Fig. 4 for $(A, \omega)$ $\approx(0.987,0.103)$ lies very close to the unrealistic region of parameter space. Small variations in one or more of the other model parameters could move this point out of the realistic region. This, however, would not remove the global bifurcations associated with the codimension-2 bifurcation point, but in the experimental situation (assuming that the model accurately describes the experimental system), the global bifurcations would seem to arise out of nowhere because only the region $A \leqslant 1$ could be investigated. We do not have experimental evidence for global bifurcations, but the numerical investigations illustrate the important point that bifurcations may be important even if they occur in an unrealizable part of the parameter region.

Many features of the bifurcation scenario illustrated in the present analysis are qualitatively identical to those observed in the forced Brusselator ${ }^{22}$ and in the forced continuous stirred tank reactor model. ${ }^{23,24}$ Gambaudo $^{25}$ has used a combination of mathematical and numerical analyses and found the same structures in models forced near Hopfbifurcation points. We refer to those papers for more detailed descriptions of the global bifurcations as well as of the solutions in the different regions.

In conclusion, we have presented clinical experimental and numerical evidence of phase-locking and other modes in human insulin secretion stimulated with oscillatory glucose infusion. Further clinical studies performed in conjunction with appropriate modifications and extensions of the model could improve our insight into some aspects of in vivo regulation of insulin secretion.

\section{ACKNOWLEDGMENTS}

Supported in part by National Institutes of Health Grant Nos. DK-41814, DK-31842, DK-13941, DK-20595 (Diabetes Research and Training Center) and RR-00055 (Clinical Research Center) and by a NATO Scientific Exchange Programme-Collaborative Research Grant. Dr. Sturis is the recipient of a Research Career Development Award from the Juvenile Diabetes Foundation International.

\footnotetext{
${ }^{1}$ C. Simon, G. Brandenberger, and M. Follenius, "Ultradian oscillations of plasma glucose, insulin, and C-peptide in man during continuous enteral nutrition," J. Clin. Endocrinol. Metab. 64, 669-674 (1987).

${ }^{2}$ K. S. Polonsky, B. D. Given, and E. Van Cauter, "Twenty-four-hour profiles and pulsatile patterns of insulin secretion in normal and obese subjects," J. Clin. Invest. 81, 442-448 (1988).

${ }^{3}$ E. T. Shapiro, H. Tillil, K. S. Polonsky, V. S. Fang, A. H. Rubenstein, and E. Van Cauter, "Oscillations in insulin secretion during constant glucose
} 
infusion in normal man: relationship to changes in plasma glucose," $\mathrm{J}$. Clin. Endocrinol. Metab. 67, 307-314 (1988).

${ }^{4}$ D. A. Lang, D. R. Matthews, M. Burnett, and R. C. Tumer, "Cyclic oscillations of basal plasma glucose and insulin concentrations in human beings," N. Engl. J. Med. 301, 1023-1027 (1979).

${ }^{5}$ J. I. Stagner, E. Samols, and G. C. Weir, "Sustained oscillations of insulin, glucagon, and somatostatin from the isolated canine pancreas during exposure to a constant glucose concentration," J. Clin. Invest. 65, 939-942 (1980).

${ }^{6}$ H. F. Chou and E. Ipp, "Pulsatile insulin secretion in isolated rat islets," Diabetes 39, 112-117 (1990).

${ }^{7}$ J. Sturis, K. S. Polonsky, E. Mosekilde, and E. Van Cauter, "Computer model for mechanisms underlying ultradian oscillations of insulin and glucose," Am. J. Physiol. 260 (Endocrinol. Metab. 23), E801-E809 (1991).

${ }^{8}$ J. Sturis, E. Van Cauter, J. D. Blackman, and K. S. Polonsky, "Entrainment of pulsatile insulin secretion by oscillatory glucose infusion," J. Clin. Invest. 87, 439-445 (1991).

${ }^{9}$ J. Sturis, K. S. Polonsky, J. D. Blackman, C. Knudsen, E. Mosekilde, and E. Van Cauter, "Aspects of oscillatory insulin secretion," in Complexity, Chaos, and Biological Evolution, edited by E. Mosekilde and Li. Mosekilde (Plenum, New York, 1991), pp. 75-94.

${ }^{10} \mathrm{~L}$. Glass, M. R. Guevara, J. Belair, and A. Shrier, "Global bifurcations of a periodically forced biological oscillator," Phys. Rev. A 29, 1348-1357 (1984).

${ }^{11} \mathrm{G}$. A. Petrillo and L. Glass, "A theory for phase locking of respiration in cats to a mechanical ventilator," Am. J. Physiol. 246 (Regulatory Integrative Comp. Physiol. 15), R311-R320 (1984).

${ }^{12}$ G. Matsumoto, K. Aihara, X. Hanyu, N. Takahashi, S. Yoshizawa, and J. Nagumo, "Chaos and phase locking in normal squid axons," Phys. Lett. A 123, 162-166 (1987)

${ }^{13}$ C. Morgan and A. Lazarow, "Immunoassay of insulin: two antibody system: plasma insulin levels of normal, subdiabetic and diabetic rats," Diabetes 12, 115-126 (1963).

${ }^{14}$ O. K. Faber, C. Binder, J. Markussen, L. G. Heding, Y. K. Naithani, H. Kuzuya, P. Blix, D. L. Horwitz, and A. H. Rubenstein, "Characterization of seven C-peptide antisera," Diabetes 27 (suppl. 1), 170-177 (1978).

${ }^{15}$ R. P. Eaton, R. C. Allen, D. S. Schade, K. M. Erickson, and J. Standefer, "Prehepatic insulin production in man: kinetic analysis using peripheral connecting peptide behavior," J. Clin. Endocrinol. Metab. 51, 520-528 (1980).

${ }^{16}$ K. S. Polonsky, J. Licinio-Paixao, B. D. Given, W. Pugh, P. Rue, J. Galloway, and B. Frank, "Use of biosynthetic human C-peptide in the measurement of insulin secretion rates in normal volunteers and type I diabetic patients," J. Clin. Invest. 77, 98-105 (1986).

${ }^{17}$ E. Van Cauter, F. Mestrez, J. Sturis, and K. S. Polonsky, "Estimation of insulin secretion rates from C-peptide levels: comparison of individual and standard kinetic parameters for C-peptide clearance," Diabetes 41 , 368-377 (1992).

${ }^{18} \mathrm{C}$. Kaas-Petersen, PATH-User's Guide (University of Leeds, Leeds, UK, 1987).

${ }^{19}$ E. Van Cauter, J. D. Blackman, D. Roland, J.-P. Spire, S. Refetoff, and K. S. Polonsky, "Modulation of glucose regulation and insulin secretion by circadian thythmicity and sleep," J. Clin. Invest. 88, 934-942 (1991).

${ }^{20} \mathrm{M}$. Byrne, J. Sturis, J. D. Blackman, K. S. Polonsky, and E. Van Cauter, "Decreased glucose tolerance during sleep may be partially mediated by GH, 74th Annual Meeting of the Endocrine Society, San Antonio, Texas, 1992, Abstract No. 40.

${ }^{21}$ N. M. O'Meara, J. Sturis, E. Van Cauter, and K. S. Polonsky, "Lack of control by glucose of ultradian insulin secretory oscillations in impaired glucose tolerance and in non-insulin-dependent diabetes mellitus," J. Clin. Invest. 92, 262-271 (1993).

${ }^{22} \mathrm{C}$. Knudsen, J. Sturis, and J. S. Thomsen, "Generic bifurcation structures of Arnol'd tongues in forced oscillators," Phys. Rev. A 44, 3503-3510 (1991).

${ }^{23} \mathrm{~W}$. Vance and $\mathrm{J}$. Ross, "A detailed study of a forced chemical oscillator: Arnol'd tongues and bifurcation sets," J. Chem. Phys. 91, 7654-7670 (1989).

${ }^{24}$ M. A. Taylor and I. G. Kevrekidis, "Some common dynamic features of coupled reacting systems," Physica D 51, 274-292 (1991).

${ }^{25} \mathrm{~J}$. M. Gambaudo, "Perturbation of a Hopf bifurcation by an external timeperiodic forcing," J. Differential Eqs. 57, 172-199 (1985). 